\title{
Thromboprophylaxis - A Neglected Front in Hospitalised Patients with COVID-19 in Pakistan
}

\author{
Mohammad Ammad Ud Din, ${ }^{1}$ Abdul Basit Shah Vardag² and Faiz Anwer ${ }^{3}$ \\ 1. Department of Internal Medicine, Rochester General Hospital, Rochester, NY, USA; 2. Department of Otorhinolaryngology, \\ Aga Khan University, Karachi, Pakistan; 3. Department of Hematology/Oncology, Cleveland Clinic, Cleveland, OH, USA
}

$\mathrm{T}$ he COVID-19 pandemic has presented a unique challenge, testing healthcare systems at a global level. The primary objective of healthcare facilities in these dire circumstances has been to treat the respiratory failure associated with viral pneumonia. However, a growing amount of literature has started to emphasise the importance of a well-rounded approach and has shed light on the various other manifestations of the disease. Amongst these significant manifestations is the risk of thrombotic events and the need for appropriate thromboprophylaxis in hospitalised patients in order to reduce patient morbidity and mortality. However, in developing nations such as Pakistan, where there has been striking lack of basic healthcare provisions at government facilities, the necessity of thromboprophylaxis is currently being overlooked.

\section{Keywords}

COVID-19, coronavirus, thromboprophylaxis, heparin, anticoagulation

Disclosures: Mohammad Ammad Ud Din, Abdul Basit Shah Vardag and Faiz Anwer have no financial or non-financial relationships or activities to declare in relation to this article.

Review Process: Double-blind peer review. Compliance with Ethics: This article is an opinion piece and does not report on new clinical data, or any studies with human or animal subjects performed by any of the authors.

Authorship: The named authors meet the International Committee of Medical Journal Editors (ICMJE) criteria for authorship of this manuscript, take responsibility for the integrity of the work as a whole, and have given final approval for the version to be published. Access: This article is freely accessible at touchONCOLOGY.com (c) Touch Medical Media 2020. Received: 14 May 2020

Accepted: 26 June 2020

Published Online: 16 July 2020

Citation: European Oncology \& Haematology. 2020;16(1):14-5

Corresponding Author: Mohammad Ammad Ud Din, Department of Internal Medicine, Rochester General Hospital, 1425 Portland Avenue Rochester, New York, NY 14621, USA. E: ammadahr@gmail.com

Support: No funding was received in the publication of this article.
As of June 2020, the COVID-19 pandemic has swept across 188 countries, infecting more than 10 million people worldwide. ${ }^{1}$ Despite its proximity to China, Pakistan's death toll from the virus, as of the end of June 2020, has fortunately remained under 5,$000 ;{ }^{1}$ however, the reported number of cases continues to increase and the death toll is estimated to exceed 42,000 by October 2020, ${ }^{2}$ in spite of the government's efforts to curb the spread by implementing lockdowns and promoting social distancing measures. As more data emerge concerning the various manifestations of the illness, guidelines regarding the management of hospitalised patients continue to change to ensure comprehensive patient care on all fronts. Although the emphasis is largely on the management of respiratory failure and in the search for targeted therapy against the virus, various multicentre studies have reported an increased incidence of thrombotic events in hospitalised patients with severe acute respiratory syndrome coronavirus-2 (SARS-CoV-2) infection, calling for an escalated dose of thromboprophylaxis in this subset of patients.

Despite venous thromboembolism (VTE) being one of the leading causes of morbidity and mortality in hospitalised patients, apart from at a few major private centres, historically, the administration of thromboprophylaxis is not a routine part of admission orders in most hospitals in Pakistan. ${ }^{3}$ Numerous clinical trials over the past three decades have shown that the use of unfractionated heparin and low-molecular-weight heparin is safe and cost effective in reducing the risk of deep-vein and pulmonary embolism in hospitalised patients. ${ }^{4}$ Based on these studies, weight-based thromboprophylaxis has widely been adopted as a part of the general admission order set in Europe and North America in adult patients who do not have increased bleeding risk and are expected to be immobile for the majority of their hospital stay. Furthermore, in patients with a higher risk of thrombotic events, such as post-orthopaedic or bariatric surgery, an escalated dose of thromboprophylaxis is preferred. This practice, for logistical reasons or otherwise, is generally not undertaken in the majority of Pakistani hospitals because of multiple barriers to high-quality patient care. Important factors to consider are poor socioeconomic conditions and the absence of comprehensive health insurance programmes at a national level. ${ }^{5,6} \mathrm{~A}$ major portion of the annual healthcare budget is spent to provide medical services at a subsidised cost in public hospitals, ${ }^{6}$ and due to lack of financial resources, many admission orders that are essential in the West, including thromboprophylaxis, are not considered cost-effective and hence are generally not implemented.

Similarly, despite global recognition, the burden of disease from thromboembolic events for patients with COVID-19 has not been fully realised by healthcare facilities in Pakistan, which may lead to increased mortality in hospitalised patients with COVID-19. Studies are suggesting that the risk of a thrombotic event may be as high as $25-30 \%$ in patients with severe infection, particularly those who require admission into the intensive care unit (ICU) because of acute respiratory distress syndrome (ARDS).? 
Several factors synergistically contribute to making patients with severe SARS-COV-2 infection more prone to both arterial embolisms and VTES. The evidence of coagulopathy is indicated by markedly increased levels of D-dimers amongst patients and an erratic pattern of deranged prothrombin time and other coagulation indicators. ${ }^{8}$ Moreover, it is also theorised that the virus can directly or indirectly cause damage to the endothelial cells lining the small pulmonary vessels. ${ }^{9}$ This may explain why patients with diabetes and hypertension are at a higher risk of severe disease, as the endothelial lining of blood vessels is already compromised in such individuals. The damaged endothelial cells then initiate the coagulation cascade, creating a prothrombotic microenvironment. ${ }^{9}$ The isolation of patients in confined places leads to venous stasis due to immobilisation, and as an added insult is seen in critically ill patients in the ICU who are sedated and on mechanical ventilation. Secondly, the state of impaired pulmonary gas exchange and severe pulmonary inflammation results in hypoxia from acute lung injury, which in turn leads to the activation of endothelial cells and macrophages, causing a surge in various pro-inflammatory interleukins and tumour necrosis factor-alpha, forming a cytokine storm-like condition, aiding coagulation. ${ }^{4}$ Moreover, patients with COVID-19 who progress to develop ARDS are usually elderly and their baseline advanced age-related morbidities put them at a greater risk for VTE. ${ }^{8} \mathrm{~A}$ large multicentre study by Wang et al. of over 1,000 hospitalised patients with COVID-19 showed that $40 \%$ of them were at high risk of VTE. Eleven per cent of the high-risk patients who were not on thromboprophylaxis developed a VTE during the hospital stay. ${ }^{10}$

Similarly, a study by Tang et al. of nearly 450 patients with COVID-19 demonstrated that, among the subset of patients with a sepsis-induced coagulopathy score $\geq 4$ or D-dimer $\geq 6$ times the normal upper limit, those who were on low-molecular-weight heparin had a significantly lower 28-day mortality rate compared with those not on anticoagulation. ${ }^{11}$ Klok et al. evaluated 184 patients in ICU with COVID-19, all of whom received at least the standard weight-based thromboprophylaxis.
Of these patients, only those with a clinical suspicion of VTE, such as leg swelling and/or acute hypoxia, underwent diagnostic imaging for further evaluation. The results showed nearly $30 \%$ of patients had VTE and $3.7 \%$ of patients had arterial thrombosis confirmed by imaging, indicating the need for escalated dose thromboprophylaxis in severely ill patients with COVID-19.7 Moreover, the thrombogenicity of COVID-19 has also been further elucidated by autopsy studies showing severe endothelial injury with alveolar capillary microthrombi and angiogenesis in patients with severe disease. ${ }^{12}$

Based on these studies, VTE prophylaxis for patients with COVID-19 has been formally incorporated into different international guidelines, such as the Swiss consensus statement ${ }^{13}$ and the United Kingdom's National Health Service (NHS) model..$^{14}$ The American Society of Hematology also supports the use of thromboprophylaxis in these patients. ${ }^{15}$ Most centres in high-income countries have formed their anticoagulation algorithms for treatment of patients with COVID-19, aiming for escalated dose thromboprophylaxis in patients with markedly deranged inflammatory markers, e.g. D-dimer $\geq 3$ times the normal upper limit, or patients requiring ICU admission; with some centres using full-dose anticoagulation in cases with a high suspicion of thrombotic event, where imaging cannot be performed to confirm the diagnosis.

The routine use of thromboprophylaxis in the management of patients with COVID-19 in hospitals across Pakistan may lead to added costs associated with the use of unfractionated heparin/low-molecular-weight heparin and imaging for VTE, which can increase the financial burden on the healthcare system, especially in the government sector. However, it will help lower the mortality rate and possibly shorten hospital stays. The lack of advanced treatment options, such as catheter-associated thrombolysis and extracorporeal membranous oxygenation for massive pulmonary embolism, further justifies the use of routine thromboprophylaxis in hospitalised patients as part of good standard practice.
1. John Hopkins University \& Medicine. Coronavirus Resource Center. COVID-19 Dashboard by the Center for Systems Science and Engineering (CSSE) at Johns Hopkins University (JHU). 2020. Available at: https://coronavirus.jhu.edu/man htm (accessed 13 July 2020)

2. Institute for Health Metrics and Evaluation. New IHME COVID-19 forecasts predict more than 40,000 deaths in Pakistan by October 1.26 June 2020. Available at: www.healthdata.org/news-release/new-ihme-covid-19whw.healthdata.org/news-release/new-ihme-covid-19-
forecasts-predict-more-40000-deaths-pakistan-october-1 (accessed 13 July 2020)

3. Khan AA, Zafar SN. Venous thromboembolism in Pakistan: a neglected research agenda. J Thromb Thrombolysis. 2013;35:234-42

4. Kahn SR, Morrison DR, Cohen JM, et al. Interventions for implementation of thromboprophylaxis in hospitalized medica and surgical patients at risk for venous thromboembolism. Cochrane Database Syst Rev. 2013:CD008201.

5. Ali NS, Nawaz A, Junaid M, et al Venous thromboembolism - incidence of deep venous thrombosis and pulmonary embolism in patients with head and neck cancer: a tertiary care experience in Pakistan. Int Arch Otorhinolaryngol. 2015;19:200-4.

6. Hassan A, Mahmood K, Bukhsh HA. Healthcare system of Pakistan. IJARP. 2017:1:170-3

7. Klok FA, Kruip MJ, Van der Meer NJ, et al. Incidence of thrombotic complications in critically ill ICU patients with COVID-19. Thromb Res. 2020:191:145-7.

8. Xu JF, Wang $L$, Zhao $L$, et al. Risk assessment of venous thromboembolism and bleeding in COVID-19 patients. Resp Res. 24 March 2020; PREPRINT (Version 1) DOl: 10.21203/rs.3.rs-18340/v1

9. Matacic C. Blood vessel injury may spur disease's fatal second phase. Science. 2020;368:1039-40

10. Wang T, Chen R, Liu C, et al. Attention should be paid to venous thromboembolism prophylaxis in the management of COVID-19. Lancet Haematol. 2020;7:e362-3.

11. Tang $\mathrm{N}$, Bai $\mathrm{H}$, Chen $\mathrm{X}$, et al. Anticoagulant treatment is associated with decreased mortality in severe coronavirus disease 2019 patients with coagulopathy. J Thromb Haemost. 2020;18:1094-9.

12. Ackermann $\mathrm{M}$, Verleden $\mathrm{SE}$, Kuehnel $\mathrm{M}$, et al. Pulmonary vascular endothelialitis, thrombosis, and angiogenesis in Covid-19. N Eng J Med. 2020; 383:120-8.

13. Casini A, Alberio L, Angelillo-Scherrer A, et al.

Thromboprophylaxis and laboratory monitoring for in-hospital patients with COVID-19 - a Swiss consensus in-hospital patients with COVID-19 - a Swiss consensus statement by the Working Party Hemostasis. Swiss Med Wkly. 2020;150:w20247.

14. NHS. Coronavirus guidance for clinicians and NHS managers. 2020. Available at: www.england.nhs.uk/coronavirus/ (accessed 30 June 2020).

15. American Society of Hematology (ASH). COVID-19 Resources. COVID-19 and VTE-anticoagulation. 2020. Available at: www.hematology.org/covid-19/covid-19-and-vteanticoagulation (accessed 13 July 2020). 